\title{
Chronic respiratory disease surveys in adults in low- and middle-income countries: A systematic scoping review of methodological approaches and outcomes
}

\author{
Nik Sherina Hanafi ${ }^{*}$, Dhiraj Agarwal2*, \\ Soumya Chippagiri ${ }^{3}$, Evelyn A \\ Brakema ${ }^{4}$, Hilary Pinnock ${ }^{5}$, Aziz \\ Sheikh ${ }^{5}$, Su-May Liew ${ }^{1}$, Chiu-Wan $\mathrm{Ng}^{6}$, \\ Rita Isaac ${ }^{3}$, Karuthan Chinna ${ }^{7}$, Li Ping \\ Wong ${ }^{6}$, Norita Hussein ${ }^{1}$, Ahmad Ihsan \\ Abu Bakar ${ }^{8}$, Yong-Kek Pang ${ }^{9}$, Sanjay \\ Juvekar², Ee Ming Khoo'; RESPIRE \\ Collaborators
}

\footnotetext{
${ }^{1}$ Department of Primary Care Medicine, Faculty of Medicine, University of Malaya, Kuala Lumpur, Malaysia

${ }^{2}$ KEM Hospital Research Centre, Pune, India

${ }^{3}$ Christian Medical College, Vellore, India

${ }^{4}$ Department of Public Health and Primary Care, Leiden University Medical Centre, Leiden, the Netherlands

${ }^{5}$ NIHR Global Health Research Unit on Respiratory Health (RESPIRE), Usher Institute, The University of Edinburgh, Edinburgh, UK

${ }^{6}$ Department of Social and Preventive Medicine, Faculty of Medicine, University of Malaya, Kuala Lumpur, Malaysia

${ }^{7}$ School of Medicine, Faculty of Health and Medical Sciences, Taylor's University, Subang Jaya, Malaysia ${ }^{8}$ Pusrawi Hospital Sdn Bhd, Kuala Lumpur, Malaysia

${ }^{9}$ Department of Medicine, Faculty of Medicine, University of Malaya, Kuala Lumpur, Malaysia *These authors contributed equally.
}

\section{Correspondence to:}

Ee Ming Khoo, MBBS (London), MD (Mal),

MRCGP(UK), FAMM, FAFP

Department of Primary Care Medicine

Faculty of Medicine

University of Malaya

50603 Kuala Lumpur

Malaysia

khooem@um.edu.my
Background Chronic respiratory diseases (CRDs) contribute significantly towards the global burden of disease, but the true prevalence and burden of these conditions in adults is unknown in the majority of low- and middle-income countries (LMICs). We aimed to identify strategies - in particular the definitions, study designs, sampling frames, instruments, and outcomes - used to conduct prevalence surveys for CRDs in LMICs. The findings will inform a future RESPIRE Four Country ChrOnic Respiratory Disease (4CCORD) study, which will estimate CRD prevalence, including disease burden, in adults in LMICs.

Methods We conducted a scoping review to map prevalence surveys conducted in LMICs published between 1995 and 2018. We followed Arksey and O'Malley's six-step framework. The search was conducted in OVID Medline, EMBASE, ISI Web of Science, Global Health, WHO Global Index Medicus and included three domains: CRDs, prevalence and LMICs. After an initial title sift, eight trained reviewers undertook duplicate study selection and data extraction. We charted: country and populations, random sampling strategies, CRD definitions/phenotypes, survey procedure (questionnaires, spirometry, tests), outcomes and assessment of individual, societal and health service burden of disease.

Results Of 36872 citations, 281 articles were included: 132 from Asia (41 from China). Study designs were cross-sectional surveys $(n=260)$, cohort studies $(\mathrm{n}=11)$ and secondary data analysis $(\mathrm{n}=10)$. The number of respondents in these studies ranged from 50 to 512891 . Asthma was studied in 144 studies, chronic obstructive pulmonary disease (COPD) in 112. Most studies (100/144) based identification of asthma on symptom-based questionnaires. In contrast, COPD diagnosis was typically based on spirometry findings (94/112); 65 used fixed-ratio thresholds, 29 reported fixed-ratio and lower-limit-of-normal values. Only five articles used the term 'phenotype'. Most studies used questionnaires derived from validated surveys, most commonly the European Community Respiratory Health Survey $(\mathrm{n}=47)$. The burden/impact of CRD was reported in 33 articles (most commonly activity limitation).

Conclusion Surveys remain the most practical approach for estimating prevalence of $C R D$ but there is a need to identify the most predictive questions for diagnosing asthma and to standardise diagnostic criteria. 
Chronic respiratory diseases (CRDs) contribute significantly to the national, regional and global burden of disease [1]. Although the morbidity and mortality of such conditions are estimated to be high in low- and middle-income countries (LMICs), there is concern that there is little robust data on the true prevalence of asthma and chronic obstructive pulmonary disease (COPD) in these countries [2,3]. Accurate data on prevalence, including disease burden, are needed to inform health care policy on prioritising care and targeting risk factors particularly for conditions that are preventable and treatable in primary care.

CRD encompasses several conditions. Most common are asthma and COPD; but others such as bronchiectasis, post-tuberculosis, interstitial lung disease and lung cancer are potentially important causes of morbidity in LMICs. In poorly-resourced primary health care systems in LMICs the conditions often remain undiagnosed as limited access to health care is compounded by insufficient attention to the conditions and lack of diagnostic capabilities in the facilities $[4,5]$. Determining the prevalence of asthma and COPD in the community remains a challenge because of the poor sensitivity and specificity of widely used questionnaire-based research tools $[3,6]$. Objective testing with spirometry may be a challenge in community-based epidemiological surveys [7], particularly in LMICs.

Scoping reviews are used to map the literature available and to identify potential gaps in the evidence base [8]. We conducted a systematic scoping review on the prevalence of CRDs to address the following questions with respect to prevalence studies on CRDs:

1. What surveys on the prevalence of asthma, COPD and other CRDs have been undertaken in LMICs?

2. What definitions, questionnaires, tests, diagnostic processes and outcomes for CRDs did the surveys employ?

3. How was the socio-economic burden (from a societal or healthcare perspective) of asthma, COPD and other CRDs estimated in these surveys?

4. What strategies have been used to identify phenotypes of asthma and COPD, or to identify the causes of 'other CRD'?

We intend to use the findings of this scoping review to inform the methodology of a proposed RESPIRE survey in 4 Countries estimating prevalence and burden of Chronic Respiratory Disease in adults in LMICs [4CCORD study].

\section{METHODS}

We followed the six-step framework for undertaking scoping reviews described by Arksey and O'Malley [9] which has been widely employed $[10,11]$. The full protocol for this systematic scoping review has been published [12]. The research questions and methods are summarised below.

\section{Search strategy}

We developed a comprehensive search strategy assisted by information librarians. The search strategy (MEDLINE search strategy detailed in Table S1 in the Online Supplementary Document) comprised of keywords and subject headings (eg, MeSH) that were used to identify studies within three domains - CRDs, prevalence (including 'burden' or 'cost' in the title/abstract) and LMICs. The strategy was first conducted in OVID MEDLINE. We then tailored and applied the strategy in EMBASE, ISI WoS, Global Health and WHO Global Index Medicus. We included studies published between 1995, when the Global Initiative on Asthma (GINA) was launched [13], and 2018. We did not limit our search to any language.

\section{Study selection}

We included articles reporting community-based prevalence studies of CRDs that were published in academic journals. Eight members of the research team were involved in the initial study selection. As a scoping review, our search criteria were sensitive rather than specific. We anticipated many hits, so we carried out the selection process in three stages:

- A single reviewer performed initial title sift to exclude obviously irrelevant titles. Two reviewers (DA, $\mathrm{NSH}$ ) conducted this individually on separate parts of the dataset.

- Screening of titles and abstracts (DA and NSH worked on separate datasets)

- Full text screening of potentially relevant articles (DA, NSH, HP, SC, SS or EAB) 
Table 1. Inclusion and exclusion criteria [12].

$\begin{array}{ll}\text { CRITERION } & \text { INCLUSION AND EXCLUSION CRITERIA } \\ \text { Population } & \text { We included surveys on general populations of adults (typically } \\ & \geq 18 \text { years but used different thresholds according to age of ma- } \\ & \text { jority which may vary in different countries). Surveys that includ- } \\ & \text { ed both adults and children were included but those that focused } \\ & \text { entirely on children were excluded. We excluded surveys on pop- } \\ & \text { ulations with known CRDs or respiratory diseases symptoms (for } \\ & \text { example: attendees at a respiratory clinic). }\end{array}$

To ensure better understanding of the studies' methodological framework, at each stage we undertook a training process of duplicate screening of 200 citations. We compared initial decisions, resolved disagreements, and refined the inclusion and exclusion criteria in discussion with three arbiters (HP, EMK and SJ). This was repeated until we were satisfied that the selection criteria were clear, and researchers were achieving at least 90\% agreement. At all stages, articles were grouped according to their relevance (relevant, not relevant or unsure); researchers were instructed to use an 'unsure' category if there was any doubt about relevance and these articles were then discussed within the team and a decision made. The selection criteria and definitions applied are detailed in Table 1 [12].

\section{Data charting}

In line with scoping review methodology, we did not assess the quality of the individual studies because we aimed to map the evidence and not to summarise the study results nor to analyse disease risks and prevalence rates. We piloted and refined a customised data extraction form. Six reviewers (DA, NSH, SC, SS, HP or EAB) independently extracted data into the extraction form. Any ambiguities were resolved within the group. Studies from the same study population were reported as independent studies, with reference to the main article if indicated. Our research questions focused on identifying the process (rather than the outcomes) of undertaking surveys of CRD in LMICs. We made one attempt to contact authors of included articles for additional information, copies of questionnaires or study procedures if they were not otherwise available.

\section{Summarising and reporting the results}

We summarised the data focusing on: 1) the strategies used to identify randomly sampled populations in LMICs; 2) the disease definitions used (which may vary over time); 3) the questionnaires used and tests performed to detect asthma, COPD and/or other CRDs; 4) the individual, societal and health care burden of CRD, and the risk factors for disease and 5) surveys that addressed contemporary understanding of asthma/COPD phenotypes. We held an investigators' meeting with RESPIRE partners (from Bangladesh, India, Malaysia, Pakistan and Edinburgh, UK), where we discussed the findings. We followed the Preferred Reporting Items for Systematic reviews and Meta-Analyses extension for. Scoping Reviews (PRISMA-ScR) [17].

\section{RESULTS}

The literature search yielded 36872 citations from five databases. After screening 729 full text articles, 281 were included in this scoping review (Figure 1). The study characteristics are detailed in Table S2 in the Online Supplementary Document.

\section{Question 1: What surveys on the prevalence of asthma, COPD and other CRDs have been undertaken in LMICs?}

The 281 publications on CRD prevalence surveys in LMCs, published between 1995 and 2018, reported studies conducted in a total of 70 countries. Most publications were based on surveys conducted in Asia ( $\mathrm{n}=133$ publications) with China, India and Turkey having 42, 35 and 30 publications, respectively 


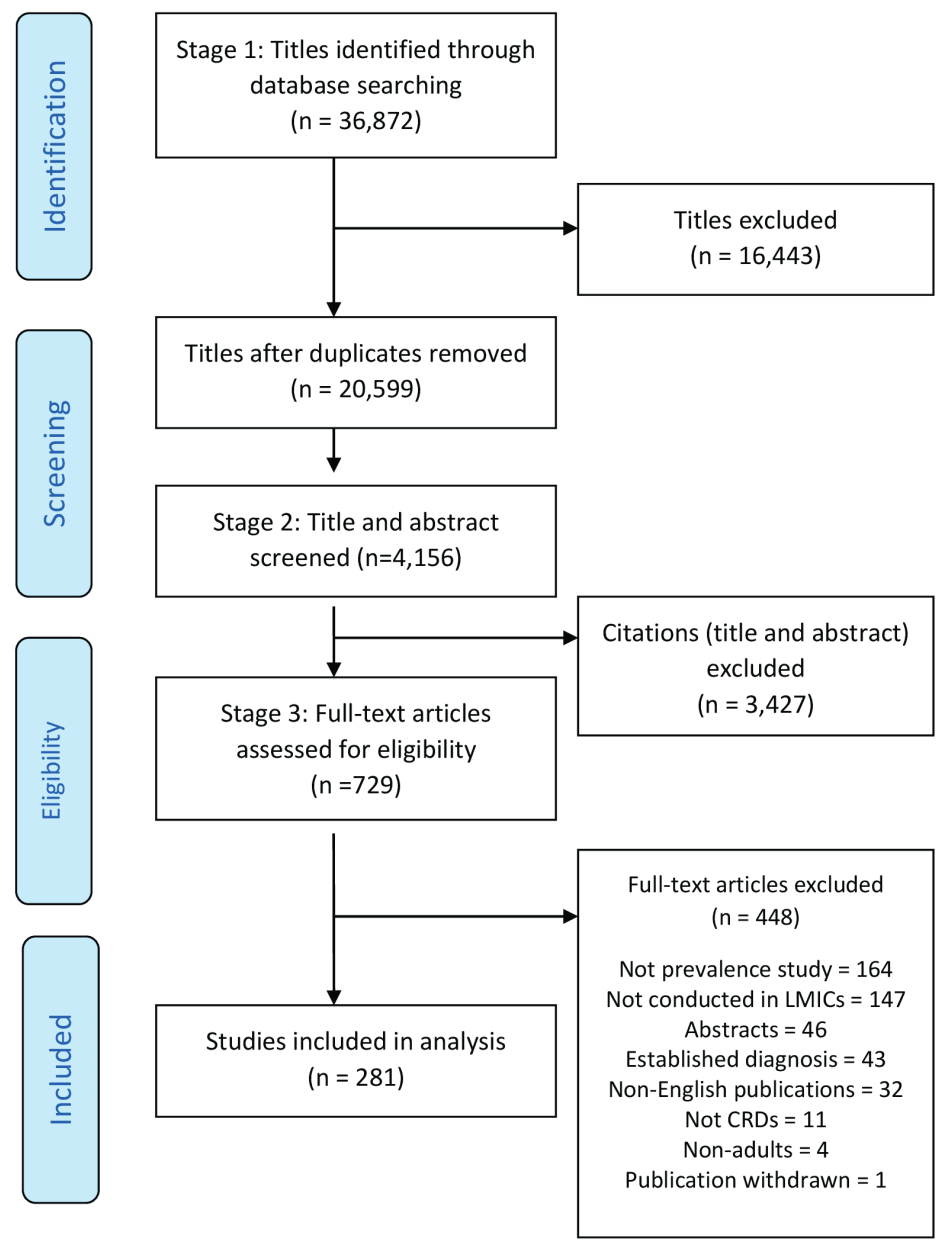

Figure 1. Preferred Reporting Items for Systematic Reviews And Meta-Analyses (PRISMA) flowchart for study selection process.

(Table S1 in the Online Supplementary Document). This is followed by publications reported from the African $(n=45)$ and European continents $(n=39)$ (Figure 2). The number of publications reporting the prevalence of CRDs increased steadily over the years, from 11 between 1995-1999 to 98 between 2015-2018. Some studies, such as the BOLD study [16], was carried out in high-income countries as well as LMICs.

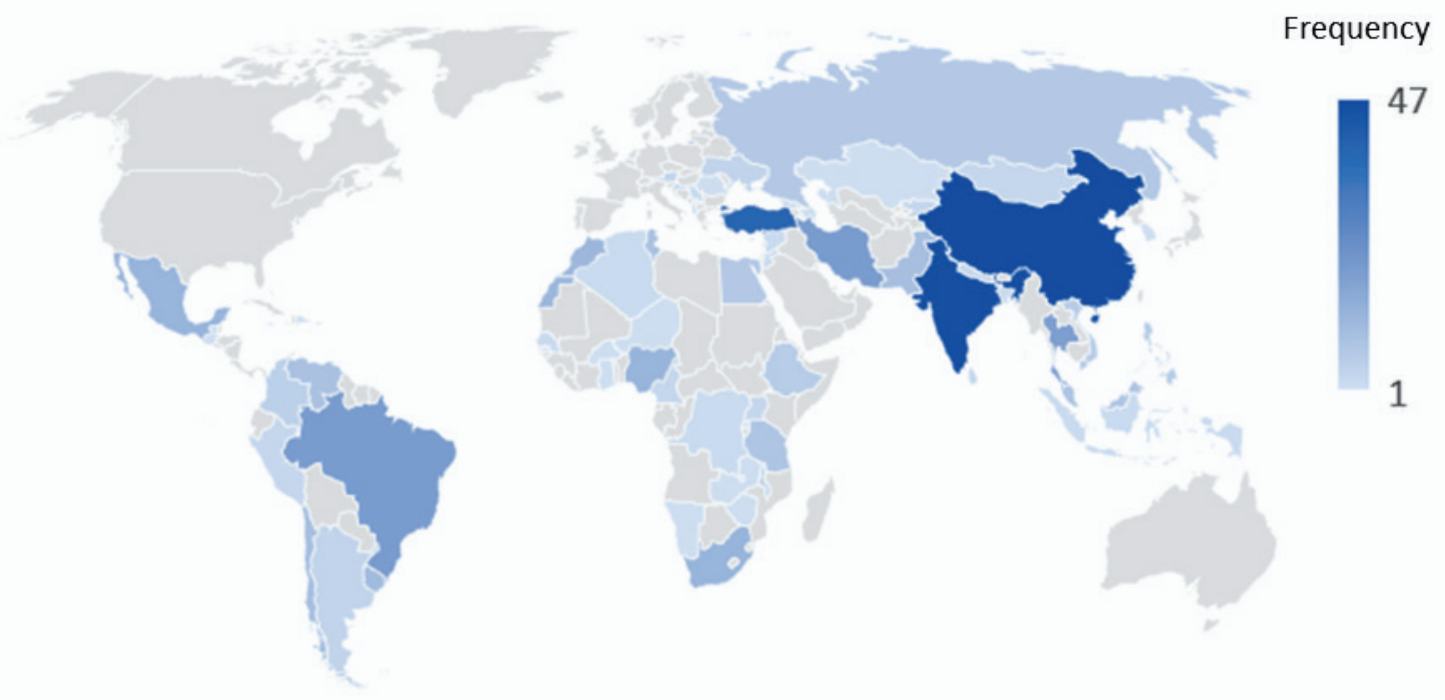

Figure 2. Distribution of chronic respiratory disease prevalence studies. 
Of the 281 publications, 181 sampled from general populations, 57 recruited specific groups (clinic attendees or staff, university students and staff, elderly, women, men, athletes, smokers, non-smokers, aboriginal people and those with family history of asthma) and 43 were based on occupational groups. In this review, only surveys that screened for asthma, COPD and/or other CRDs were included. Surveys for specific occupational lung disease were excluded. Details are in Table S2 in the Online Supplementary Document.

The study designs of the 281 studies varied: cross-sectional surveys $(n=260)$, cohort surveys $(n=11)$ and secondary data analyses $(n=10)$. The cross-sectional surveys were typically house-to-house or community surveys ( $n=178)$, at worksites $(n=40)$, surveys in clinics or health care facilities $(n=20)$, in universities $(n=8)$, telephone $(n=7)$ and postal surveys $(n=3)$; one postal survey was followed by a house-to-house survey due to initial low response rate.

The number of respondents in each study ranged from 50 to 512891 . Ten publications reported sample sizes of 100000 or more; five in India [18-22], three in China [23-25] and two in multiple countries [26,27]. 101 studies had fewer than 1000 respondents. Response rates were reported in 128 surveys, out of which 91 achieved at least $80 \%$.

\section{Question 2. What definitions, questionnaires, tests and diagnostic processes and outcomes for CRDs did the surveys employ?}

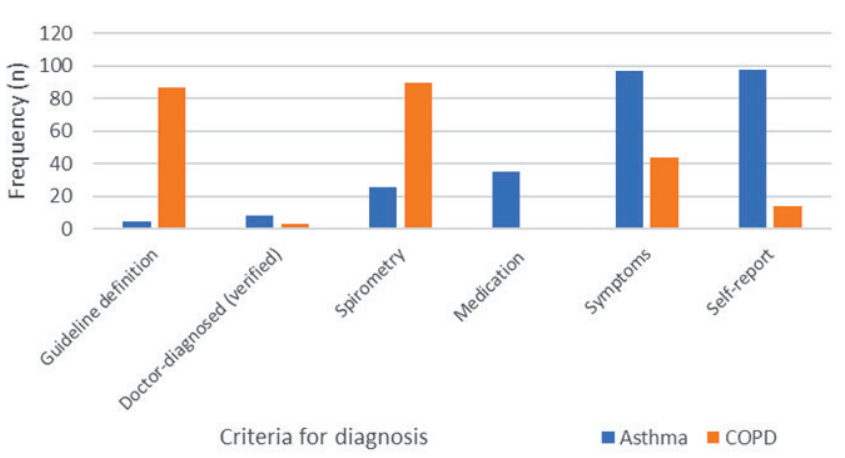

Figure 3. Criteria Used for Diagnosis of Asthma and COPD.

\section{Definition of diseases}

Surveys used various definitions for both asthma and COPD. Figure 3 contrasts the approach for asthma and COPD.

For asthma diagnosis, most surveys used self-reported diagnosis ( $\mathrm{n}=98$ ), or a questionnaire survey of symptoms $(\mathrm{n}=97)$. Other methods were use of asthma medication $(n=35)$, spirometry $(n=26)$, verification through records $(\mathrm{n}=8)$ and reference to GINA guidelines $(\mathrm{n}=5)$. These methods were sometimes used in combination.

In marked contrast, for COPD diagnosis, 90 articles based the diagnosis on spirometry. Of these, 59 were based on a fixed Forced Expiratory Volume in one second/Forced Vital Capacity (FEV1/FVC) ratios, 28 used both fixed ratios and lower limits of normal (LLN) and three used only lower limit of normal as diagnostic criteria. Lower limit of normal was used in more recent studies (published since 2008). Other methods, which were sometimes used in combination, were according to GOLD guidelines $(n=87)$, symptoms $(n=44)$, self-reported diagnosis $(n=14)$ and doctor's diagnosis, verified through records $(n=3)$.

\section{Questionnaires}

Most studies used validated questionnaires such as the European Community Respiratory Health Survey (ECRHS) ( $n=58)$, American Thoracic Society Respiratory Questionnaire (ATS) $(n=43)$, International Union against Tuberculosis and Lung Disease (IUATLD) $(\mathrm{n}=23)$ and the Medical Research Council Respiratory Questionnaire (MRC) $(n=14)$, either in totality, adapted or modified. The BOLD $(n=30)$ and PLATINO $(n=12)$ surveys used questionnaires derived from both the ECRHS and ATS questionnaires.

Common symptoms explored in the surveys were cough, wheezing, expectoration, dyspnea, breathlessness or phlegm as well nasal symptoms of itching, obstruction, sneezing, and secretion.

\section{Spirometry or peak flow meter}

Baseline spirometry was measured in 167 studies; of these, 109 also reported post-bronchodilator readings, usually to salbutamol (albuterol) in doses ranging from 100 to $400 \mu$ g. Two surveys used ipratropium and two used Combivent ${ }^{\mathrm{TM}}$. Most studies reported on spirometry findings of obstructive patterns; only 22 studies reported prevalence of restriction. One study included chest x-ray findings to measure the prevalence of pneumoconiosis [28]. Two studies carried out in Malawi and Thailand [29,30] reported prevalence of restrictive lung disease using European normal values that could be an overestimation while four studies used modified values or lower limits of normal to suit local population [31-34]. 
Peak flow was measured in 13 surveys. Of these, five measured only peak flow without doing spirometry; four were worksite surveys [35-38] and one was a community survey exploring the effects of air quality [39].

Other diagnostic tests (each reported in one study) included exhaled CO level, DNA extraction, genetic biomarkers, pollution monitoring, RAST test, rhinomanometry, residue concentrations of pesticides in urine, serum assay for $C$ pneumoniae, urine cotinine as well as water contact and fecal contaminants.

Table 2. Study outcomes

\begin{tabular}{lc}
\hline OUTCOME & NUMBER, N (\%) \\
Disease only & $187(67)$ \\
\hline Combination of disease, symptom and/or lung function & $57(21)$ \\
\hline Symptoms only & $22(8)$ \\
\hline Lung function only & $15(5)$ \\
\hline
\end{tabular}

\section{Outcomes}

Most of the studies $(n=187)$ reported the prevalence of specific disease(s), though a minority reported symptoms or lung function test (Table 2). Asthma was the most common disease studied $(n=144)$ followed by COPD $(n=112) ; 22$ articles reported the prevalence of both asthma and COPD. Asthma COPD Overlap Syndrome (ACOS) was specifically explored in two recent surveys $[40,41]$.

\section{Question 3. How was the socio-economic burden (from a societal or health care perspective) of asthma, COPD and other CRDs estimated in these surveys?}

Burden (or impact) of respiratory disease was reported in only 33 publications. The most common variable was activity limitation $(n=22)$ followed by quality of life $(n=13)$, work absenteeism $(n=12)$ and psychosocial distress ( $n=5)$. Our search strategy focussed on prevalence studies and although we included 'cost' as a word in the title or abstract, we did not use search terms for economic evaluation of the diseases. Health care utilisation, and health status were reported in 10 and 8 studies, respectively.

\section{Question 4. What strategies have been used to identify phenotypes of asthma and COPD, or to identify the causes of 'other CRD'?}

COPD was linked to either environmental factors $(n=60)$ or occupation $(n=32)$. Only five articles (four of which were published in the last 10 years) specifically used the term 'phenotype' when describing different classifications of asthma or COPD [41-45]. Articles that reported on ACOS ( $n=2)$ were published in the last three years $[40,41]$.

\section{DISCUSSION}

\section{Summary of findings}

We identified 281 publications reporting community surveys employing diverse methods for assessing the prevalence of CRDs in general populations in LMICs. The studies were conducted in 70 countries; nearly half were from Asia. Ten articles reported surveys with more than 100000 respondents; 101 studies had fewer than 1000 respondents. Surveys typically focussed on detecting either asthma (122 studies) or COPD (90 studies). 22 studies explicitly aimed to identify both conditions and very few detected 'other CRDs'. Most studies used questionnaires derived from validated surveys (most commonly the ECRHS). Detection of asthma was typically based on symptoms or self-reported diagnosis. In contrast, COPD diagnosis was based on demonstration of obstruction on spirometry (without consideration of symptoms in 55\%). The burden of CRD was reported in 33 publications, most commonly activity limitation. Only five recent articles used the term 'phenotype'.

\section{Interpretation with reference to other literature}

Prevalence estimates depend on the methods and diagnostic criteria used [46]. This was previously highlighted in a systematic review done in Europe [47] which showed that reported epidemiological estimates varied due to different methods including study designs. The most popular design used for studying disease prevalence in the community was household surveys. Postal surveys were not useful due to poor response rate [48-50], and one study found that the postal questionnaires were completed by persons other than the individual invited [48]. There was evidence that some surveys included quality measures, such as careful population-based sampling, standardised spirometry equipment, training and monitoring of spirometry technicians; over-reading of spirometry reports and strict protocols for questionnaire [16]. 
The studies in this review used a variety of operational definitions for asthma and COPD, employed a range of instruments, and modes of data collection although the increasing number of multi-country studies (eg, BOLD [16], PLATINO [51], PUMA [52], BREATHE [53]) have begun to standardize disease definitions. COPD guidelines are clear that a diagnosis of COPD relies on the demonstration of obstructive spirometry in a patient with chronic respiratory symptoms [54,55], yet 57 out of 112 studies in this review equated obstructive spirometry with COPD with no consideration of whether the patient had symptoms.

In contrast, the range of methods used to detect asthma reflects current discussions about the best way to confirm a diagnosis of asthma in clinical practice $[56,57]$. In LMICs, it has been suggested that the presence of asthma is better detected by having three components to the diagnosis, ie, presence of symptoms, reported physician-diagnosis, use of medication [26]. However, these approaches are often limited by inaccurate recall and recent studies have questioned the accuracy of physician diagnosis [58]. We found examples of all three of these approaches, but with little evidence of an emerging consensus, which makes it difficult to compare results across studies.

\section{Spirometry}

In our review, 167 surveys conducted spirometry to define airway obstruction, but variation in the conduct of the test and interpretation of results means that the findings are not comparable.

The optimal threshold for diagnosing airflow obstruction has been hotly debated for some years [46] and guidelines make different recommendations [13,54,59]. Almost all the early surveys in our review, including multinational BOLD and PLATINO studies, used a fixed ratio of $<70 \%$ as the diagnostic threshold for obstruction, aligned with global COPD guideline recommendations $[16,54]$. This however would underestimate the prevalence of COPD in young populations (for example, in the FRESH AIR study in Uganda where women exposed to biomass fuel since childhood had severe obstruction by the age of 35 [60]) and overestimate the prevalence of COPD in older populations [61-63]. The use of the LLN instead of a fixed criterion was advocated in 2004 [64], and in our review, studies conducted after 2008 started to use LLN, often reporting both. Use of LLN requires robust normal values from the relevant population which are not always available for LMIC countries [65], a challenge addressed in a study from Nigeria in which local normal values were derived from the non-smoking study population [66].

Discrepancies also arose because only two thirds of the studies used the recommended post-bronchodilator spirometry results, which could make considerable difference to the defined prevalence of airway obstruction [67]. A challenge, which is not yet reflected in the studies, is the recent change to the ATS/ERS standards requiring an inspiratory loop to assess for full inspiration to reduce the risk of mis-diagnosis of restrictive disorders [59]. This will incur the additional cost of providing filters to ensure safe practice in LMICs, many of which have endemic TB.

\section{Evidence gaps and implications for future surveys in LMICs}

Although surveys (questionnaires and lung function) remain the most practical approach for estimating prevalence of $\mathrm{CRD}$, our scoping review has identified several evidence gaps which should be considered in future CRD surveys in LMICs:

- Many surveys focused on detecting one condition (asthma or COPD) only; a few identified both, but hardly any mentioned other CRDs.

- In line with current moves to personalised medicine [68], a few recent surveys have begun to consider phenotypes of asthma and COPD, but none used FeNO, which is gaining importance in phenotyping asthma [69].

- Algorithms for making a clinical diagnosis (as opposed to recording lung function) were often not well formulated, especially for asthma where the most predictive questions for the diagnosis are not clear.

- Although respiratory symptoms were assessed in most surveys, and many of the questionnaires used included impact on work and social activities, the impact on the quality of life of individuals, or the social and economic burden were rarely reported.

\section{Strengths and limitations}

As this was a scoping review, it is useful for mapping common methodologies and identifying gaps in literature. However, no conclusions can be drawn on the quality of those studies. We focused on the methodologies used in community surveys, rather than analysing factors related to CRDs such as disease risks and prevalence rates. 
Despite an inclusive search strategy and 36872 hits, we may have missed some relevant studies. We did not include LILACS or Chinese language databases; however, our searches detected 30 and 42 studies conducted in South American countries (Argentina, Brazil, Chile, Colombia, Peru, Uruguay and Venezuela) and China respectively (Table S2 in the Online Supplementary Document). We did not impose a language limitation, planning to translate where possible. In the event, however, we were unable to arrange translation within our limited resources and 32 non-English papers were excluded. We had planned to check the Global Health Data Exchange website (http://ghdx.healthdata.org) for additional studies, but our checks for the four RESPIRE countries did not identify any new publications. We emailed thirteen authors to get full text articles, however only two authors responded.

In this review we aimed to scope surveys conducted in LMICs, but some multi-country surveys (eg, BOLD studies) took place in a number of high-income countries as well as several LMICs. Similarly, we were interested in surveys of CRD in adults, but some articles also included children. However, our interest was in the methodology used, which remained applicable. Even though we took steps to ensure that reviewers had at least $90 \%$ agreement in data extraction, individual reviewers' interpretation of methodological framework could be a source of bias. We did not search for health economic evaluations, though we included 'cost' as a search term, which limits the conclusions we can draw about socio-economic impact. Studies from the same study population were reported as independent studies; it is possible that study populations and similar processes were duplicated.

\section{CONCLUSION}

Accurate prevalence and disease burden information is important to understand the impact of CRDs on disability, quality of life, and to help influence public health care planning. Our scoping review identified several prevalence surveys for CRDs from a wide range of LMIC countries, but with substantial heterogeneity across the definitions, methodologies, instruments and types of outcomes used. Surveys remain the most practical approach for estimating the prevalence of CRD but there is a need to identify the most predictive questions for diagnosis asthma and to standardise diagnostic criteria. To reflect the true burden of CRDs in LMICs, future work should identify the wide range of conditions (not just asthma or COPD), and capture information on the burden of disease on an individual's quality of life and the societal burden.

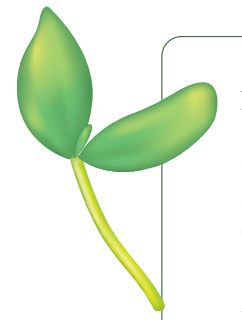

Acknowledgments: This scoping review is one of the projects within the pilot phase of the 4CCORD study. We gratefully acknowledge the advice from Dr Marshall Dozier, Academic Support Librarian, University of Edinburgh and Dr Ranita Shamsuddin, Librarian, University of Malaya for their help with developing the search strategy. We acknowledge Shalini Selvaratnam (SS), from the University of Malaya, who helped with screening articles and data extraction. We are grateful to the peer reviewers whose comments helped us refine our proposal and the RESPIRE collaborators.

Collaborators: The RESPIRE collaboration comprises the UK Grant holders, Partners and research teams as listed on the RESPIRE website (http://www.ed.ac.uk/usher/respire): Aziz Sheikh, Harry Campbell, Colin Simpson, Steve Cunningham, Monica Fletcher, Sian Williams, Liz Grant, Harish Nair, Kit Chan, Ahmed Ehsanur Rahman, Osman M Yusuf, Shahida O Yusuf, Anand Kawade, Rita Isaac, Farzana Khan, Sundeep Salvi, Deesha Ghorpade, Tabish Hazir, Sajid Bashir Soofi, Arun Dutta Roy, Muhammad Irfan.

Disclaimer: The views expressed are those of the author(s) and not necessarily those of the NHS, the NIHR or the Department of Health and Social Care.

Funding: This research was commissioned by the UK National Institute for Health Research (NIHR) Global Health Research Unit on Respiratory Health (RESPIRE), using UK Aid from the UK Government. The views expressed in this publication are those of the author(s) and not necessarily those of the NIHR or the UK Department of Health and Social Care.

Authorship contribution: SJ, EMK and HP led the development of the scoping review with DA and NSH, and with the advice of other members of the 4CCORD group. DA wrote the first draft of the protocol, which was critically reviewed and refined by SJ, EMK, HP, NSH, SC, SML, EAB and ASA. DA, NSH, EAB, HP, SC and SS conducted the scoping review and data extraction. NSH, DA, HP and KEM drafted the manuscript. NSH and DA contributed equally to the development, conduct, interpretation of findings and writing of the paper. RESPIRE UMC members and Co-Investigators of the 4CCORD study provided advice and contributed to discussions from time to time. All authors contributed to revising the manuscript critically. All authors approved the final version.

Competing interests: The authors completed the ICMJE Unified Competing Interest form (available upon request from the corresponding author), and declare no conflicts of interest.

Additional material

Online Supplementary Document 
1 Mathers CD, Loncar D. Projections of global mortality and burden of disease from 2002 to 2030. PLoS Med. 2006;3:e442. Medline:17132052 doi:10.1371/journal.pmed.0030442

2 Adeloye D, Chua S, Lee CW, Basquill C, Papana A, Theodoratou E, et al. Global and regional estimates of COPD prevalence: Systematic review and meta-analysis. J Glob Health. 2015;5:020415. Medline:26755942 doi:10.7189/jogh.05.020415

3 Salvi SS, Manap R, Beasley R. Understanding the true burden of COPD: the epidemiological challenges. Prim Care Respir J. 2012;21:249-51. Medline:22885564 doi:10.4104/pcrj.2012.00082

4 Bousquet J, Dahl R, Khaltaev N. Global alliance against chronic respiratory diseases. Eur Respir J. 2007;29:233-9. Medline:17264322 doi:10.1183/09031936.00138606

5 Lainez YB, Todd CS, Ahmadzai A, Doocy SC, Burnham G. Prevalence of respiratory symptoms and cases suspicious for tuberculosis among public health clinic patients in Afghanistan, 2005-2006: perspectives on recognition and referral of tuberculosis cases. Trop Med Int Health. 2009;14:564-70. Medline:19254273 doi:10.1111/j.1365-3156.2009.02257.x

6 Contoli M, Papi A. When asthma diagnosis becomes a challenge. Eur Respir J. 2010;36:231-3. Medline:20675777 doi:10.1183/09031936.00053010

7 Bousquet J, Dahl R, Khaltaev N. Global alliance against chronic respiratory diseases. Allergy. 2007;62:216-23. Medline:17298337 doi:10.1111/j.1398-9995.2007.01307.x

8 Peters MD, Godfrey CM, Khalil H, McInerney P, Parker D, Soares CB. Guidance for conducting systematic scoping reviews. Int J Evid-Based Healthc. 2015;13:141-6. Medline:26134548 doi:10.1097/XEB.0000000000000050

9 Arksey H, O’Malley L. Scoping studies: towards a methodological framework. Int J Soc Res Methodol. 2005;8:19-32. doi:10.1080/1364557032000119616

10 Levac D, Colquhoun H, O’Brien KK. Scoping studies: advancing the methodology. Implement Sci. 2010;5:69. Medline:20854677 doi:10.1186/1748-5908-5-69

11 Colquhoun HL, Levac D, O’Brien KK, Straus S, Tricco AC, Perrier L, et al. Scoping reviews: time for clarity in definition, methods, and reporting. J Clin Epidemiol. 2014;67:1291-4. Medline:25034198 doi:10.1016/j.jclinepi.2014.03.013

12 Agarwal D, Hanafi NS, Chippagiri S, Brakema EA, Pinnock H, Khoo EM, et al. Systematic scoping review protocol of methodologies of chronic respiratory disease surveys in low/middle-income countries. NPJ Prim Care Respir Med. 2019;29:17. Medline:31068586 doi:10.1038/s41533-019-0129-7

13 Bateman ED, Hurd S, Barnes P, Bousquet J, Drazen J, FitzGerald M, et al. Global strategy for asthma management and prevention: GINA executive summary. Eur Respir J. 2008;31:143-78. Medline:18166595 doi:10.1183/09031936.00138707

14 Rahhal B, Rmileh JA, Murrar S. Assessment of Respiratory Health Indicators among Agricultural Workers Exposed to Pesticides: A Cross Sectional Study from Palestine. Mor J Chem. 2017;5:266-71.

15 Vogelmeier CF, Criner GJ, Martinez FJ, Anzueto A, Barnes PJ, Bourbeau J, et al. Global strategy for the diagnosis, management, and prevention of chronic obstructive lung disease 2017 report. GOLD executive summary. Am J Respir Crit Care Med. 2017;195:557-82. Medline:28128970 doi:10.1164/rccm.201701-0218PP

16 Buist AS, Vollmer WM, Sullivan SD, Weiss KB, Lee TA, Menezes AMB, et al. The Burden of Obstructive Lung Disease Initiative (BOLD): Rationale and design. COPD. 2005;2:277-83. Medline:17136954 doi:10.1081/COPD-57610

17 Tricco AC, Lillie E, Zarin W, O’Brien KK, Colquhoun H, Levac D, et al. PRISMA extension for scoping reviews (PRISMA-ScR): checklist and explanation. Ann Intern Med. 2018;169:467-73. Medline:30178033 doi:10.7326/M18-0850

18 Agrawal S. Effect of Indoor Air Pollution from Biomass and Solid Fuel Combustion on Prevalence of Self-Reported Asthma among Adult Men and Women in India: Findings from a Nationwide Large-Scale Cross-Sectional Survey. J Asthma. 2012;49:355-65. Medline:22397465 doi:10.3109/02770903.2012.663030

19 Jindal SK, Aggarwal AN, Gupta D, Agarwal R, Kumar R, Kaur T, et al. Indian Study on Epidemiology of Asthma, Respiratory Symptoms and Chronic Bronchitis in adults (INSEARCH). Int J Tuberc Lung Dis. 2012;16:1270-7. Medline:22871327 doi:10.5588/ijtld.12.0005

20 Guddattu V, Swathi A, Sreekumaran N. Household and Environment Factors Associated With Asthma Among Indian Women: A Multilevel Approach. J Asthma. 2010;47:407-11. Medline:20528594 doi:10.3109/02770903.2010.481343

21 Agrawal S, Pearce N, Millett C, Subramanian SV, Ebrahim S. Occupations with an increased prevalence of self-reported asthma in Indian adults. J Asthma. 2014;51:814-24. Medline:24712498 doi:10.3109/02770903.2014.913619

22 Agrawal S, Pearce N, Ebrahim S. Prevalence and risk factors for self-reported asthma in an adult Indian population: a cross-sectional survey. Int J Tuberc Lung Dis. 2013;17:275-82. Medline:23317966 doi:10.5588/ijtld.12.0438

23 Lin J, Wang WY, Chen P, Zhou X, Wan HY, Yin KS, et al. Prevalence and risk factors of asthma in mainland China: The CARE study. Respir Med. 2018;137:48-54. Medline:29605212 doi:10.1016/j.rmed.2018.02.010

24 Smith M, Li L, Augustyn M, Kurmi O, Chen J, Collins R, et al. Prevalence and correlates of airflow obstruction in 317,000 never-smokers in China. Eur Respir J. 2014;44:66-77. Medline:24603814 doi:10.1183/09031936.00152413

25 Kurmi OP, Millwood IY, Li J, Chen B, Xie K; China Kadoorie Biobank Collaborative Group, et al. COPD and its association with smoking in the Mainland China: A cross-sectional analysis of 0.5 million men and women from ten diverse areas. Int J Chron Obstruct Pulmon Dis. 2015;10:655-65. Medline:25848242

26 To T, Stanojevic S, Moores G, Gershon AS, Bateman ED, Cruz AA, et al. Global asthma prevalence in adults: findings from the cross-sectional world health survey. BMC Public Health. 2012;12:204. Medline:22429515 doi:10.1186/1471-2458-12-204

27 Sembajwe G, Cifuentes M, Tak SW, Kriebel D, Gore R, Punnett L. National income, self-reported wheezing and asthma diagnosis from the World Health Survey. Eur Respir J. 2010;35:279-86. Medline:19741032 doi:10.1183/09031936.00027509

28 Ishtiaq M, Rabnawa Z, Khan K, Khan H, Zakir S, Sarwar G, et al. Prevalance of pneumoconiosis among coal miners of Cherat, District Nowshera - Pakistan. J Postgrad Med Inst. 2014;28:139-44. 
29 Meghji J, Nadeau G, Davis KJ, Wang DL, Nyirenda MJ, Gordon SB, et al. Noncommunicable Lung Disease in Sub-Saharan Africa A Community-based Cross-Sectional Study of Adults in Urban Malawi. Am J Respir Crit Care Med. 2016;194:67-76. Medline:26788760 doi:10.1164/rccm.201509-18070C

30 Pothirat C, Phetsuk N, Liwsrisakun C, Bumroongkit C, Deesomchok A, Theerakittikul T. Major Chronic Respiratory Diseases in Chiang Mai: Prevalence, Clinical Characteristics, and Their Correlations. J Med Assoc Thai. 2016;99:1005-13. Medline:29927203

31 Zhong N, Wang C, Wao W, Chen P, Kang J, Huang S. Prevalence of chronic obstructive pulmonary disease in China: a large, population-based survey. Am J Respir Crit Care Med. 2007;176:753-60. Medline:17575095 doi:10.1164/rccm.200612-17490C

32 Siddharthan T, Grigsby MR, Goodman D, Chowdhury M, Rubinstein A, Irazola V, et al. Association between Household Air Pollution Exposure and Chronic Obstructive Pulmonary Disease Outcomes in 13 Low- and Middle-Income Country Settings. Am J Respir Crit Care Med. 2018;197:611-20. Medline:29323928 doi:10.1164/rccm.201709-18610C

33 Pan J, Xu L, Lam TH, Jiang CQ, Zhang WS, Jin YL, et al. Association of adiposity with pulmonary function in older Chinese: Guangzhou Biobank Cohort Study. Respir Med. 2017;132:102-8. Medline:29229080 doi:10.1016/j.rmed.2017.10.003

34 Arora S, Rasania SK, Bachani D, Gandhi A, Chhabra SK. Air pollution and environmental risk factors for altered lung function among adult women of an urban slum area of Delhi: A prevalence study. Lung India. 2018;35:193-8. Medline:29697074 doi:10.4103/lungindia.lungindia_263_17

35 Stephen P, Mahalakshmy T, Manju R, Laksham KB, Subramani S, Panda K, et al. High Prevalence of Chronic Respiratory Symptoms among Autorickshaw Drivers of Urban Puducherry, South India. Indian J Occup Environ Med. 2018;22:40-4. Medline:29743784 doi:10.4103/ijoem.IJOEM_163_17

36 Ahasan MR, Ahmad SA, Khan TP. Occupational exposure and respiratory illness symptoms among textile industry workers in a developing country. Appl Occup Environ Hyg. 2000;15:313-20. Medline:10701294 doi:10.1080/104732200301638

37 Deschamps F, Sow ML, Prevost A, Henry L, Lavaud F, Bernard J, et al. Prevalence of respiratory symptoms and increased specific IgE levels in West-African workers exposed to isocyanates. J Toxicol Environ Health A. 1998;54:335-42. Medline:9650570 doi:10.1080/009841098158782

38 Ory FG, Rahman FU, Katagade V, Shukla A, Burdorf A. Respiratory disorders, skin complaints, and low-back trouble among tannery workers in Kanpur, India. Am Ind Hyg Assoc J. 1997;58:740-6. Medline:9342835 doi:10.1080/15428119791012397

39 Padhi BK, Padhy PK. Assessment of Intra-urban Variability in Outdoor Air Quality and its Health Risks. Inhal Toxicol. 2008;20:973-9. Medline:18720169 doi:10.1080/08958370701866420

40 Han YY, Forno E, Canino G, Celedon JC. Psychosocial risk factors and asthma among adults in Puerto Rico. J Asthma. 2019;56:653-61. Medline:29738276 doi:10.1080/02770903.2018.1474366

41 Montes de Oca M, Victorina Lopez Varela M, Laucho-Contreras ME, Casas A, Schiavi E, Mora JC. Asthma-COPD overlap syndrome (ACOS) in primary care of four Latin America countries: The PUMA study. BMC Pulm Med. 2017;17:69. Medline:28431499 doi:10.1186/s12890-017-0414-6

42 Celedon JC, Silverman EK, Weiss ST, Wang B, Fang Z, Xu X. Application of an algorithm for the diagnosis of asthma in Chinese families: Limitations and alternatives for the phenotypic assessment of asthma in family-based genetic studies. Am J Respir Crit Care Med. 2000;162:1679-84. Medline:11069796 doi:10.1164/ajrccm.162.5.2003007

43 de Oca MM, Halbert RJ, Lopez MV, Perez-Padilla R, Tálamo C, Moreno D, et al. The chronic bronchitis phenotype in subjects with and without COPD: the PLATINO study. Eur Respir J. 2012;40:28-36. Medline:22282547 doi:10.1183/09031936.00141611

44 Baatjies R, Lopata AL, Sander I, Raulf-Heimsoth M, Bateman ED, Meijster T, et al. Determinants of asthma phenotypes in supermarket bakery workers. Eur Respir J. 2009;34:825-33. Medline:19443530 doi:10.1183/09031936.00164408

45 Singh T, Bello B, Jeebhay MF. Risk factors associated with asthma phenotypes in dental healthcare workers. Am J Ind Med. 2013;56:90-9. Medline:22473580 doi:10.1002/ajim.22041

46 Celli BR, Halbert R, Isonaka S, Schau B. Population impact of different definitions of airway obstruction. Eur Respir J. 2003;22:268-73. Medline:12952259 doi:10.1183/09031936.03.00075102

47 Atsou K, Chouaid C, Hejblum G. Variability of the chronic obstructive pulmonary disease key epidemiological data in Europe: systematic review. BMC Med. 2011;9:7. Medline:21244657 doi:10.1186/1741-7015-9-7

48 Lam HT, Ronmark E, Nguyen VT, Ekerljung L, Nguyen TKC, Lundback B. Increase in asthma and a high prevalence of bronchitis: Results from a population study among adults in urban and rural Vietnam. Respir Med. 2011;105:177-85. Medline:21030231 doi:10.1016/j.rmed.2010.10.001

49 Lai CK, Ho SC, Lau J, Yuen YK, Ho SS, Chan CH, et al. Respiratory symptoms in elderly Chinese living in Hong Kong. Eur Respir J. 1995;8:2055-61. Medline:8666100 doi:10.1183/09031936.95.08122055

50 Papageorgiou N, Gaga M, Marossis C, Reppas C, Avarlis P, Kyriakou M, et al. Prevalence of asthma and asthma-like symptoms in Athens, Greece. Respir Med. 1997;91:83-8. Medline:9122516 doi:10.1016/S0954-6111(97)90072-X

51 Caminha GP, Pizzichini E, Neto JFL, Hopkins C, Moreira JD, Pizzichini MMM. Rhinosinusitis symptoms, smoking and COPD: Prevalence and associations. Clin Otolaryngol. 2018;43:1560-5. Medline:30152142 doi:10.1111/coa.13215

52 Schiavi E, Stirbulov R, Vecino RH, Mercurio S, Di Boscio V, Equipo P. COPD Screening in Primary Care in Four Latin American Countries: Methodology of the PUMA Study. Arch Bronconeumol. 2014;50:469-74. Medline:24816036 doi:10.1016/j. arbres.2014.03.006

53 El Hasnaoui A, Rashid N, Lahlou A, Salhi H, Doble A, Nejjari C, et al. Chronic obstructive pulmonary disease in the adult population within the Middle East and North Africa region: rationale and design of the BREATHE study. Respir Med. 2012;106:S315. Medline:23290702 doi:10.1016/S0954-6111(12)70010-0

54 Global Initiative for Chronic Obstructive Lung Disease. Global Strategy for the Diagnosis, Management, and Prevention of Chronic Obstructive Pulmonary Disease Report. 2019. 
55 National Institute for Health and Care Excellence. Asthma: diagnosis, monitoring and chronic asthma management. 2017.

56 White J, Paton JY, Niven R, Pinnock HJT. Guidelines for the diagnosis and management of asthma: a look at the key differences between BTS/SIGN and NICE. Thorax. 2018;73:293-7. doi:10.1136/thoraxjnl-2017-211189

57 Daines L, McLean S, Buelo A, Lewis S, Sheikh A, Pinnock H. Clinical prediction models to support the diagnosis of asthma in primary care: a systematic review protocol. NPJ Prim Care Respir Med. 2018;28:15. Medline:29777106 doi:10.1038/s41533018-0086-6

58 Aaron SD, Boulet LP, Reddel HK. Gershon ASJAjor. Underdiagnosis and overdiagnosis of asthma. Am J Respir Crit Care Med. 2018;198:1012-20. Medline:29756989 doi:10.1164/rccm.201804-0682CI

59 Graham BL, Steenbruggen I, Miller MR, Barjaktarevic IZ, Cooper BG, Hall GL, et al. Standardization of spirometry 2019 update. An official American thoracic society and European respiratory society technical statement. Am J Resp Crit Care Med. 2019;200:e70-88. Medline:31613151 doi:10.1164/rccm.201908-1590ST

60 van Gemert F, Kirenga B, Chavannes N, Kamya M, Luzige S, Musinguzi P, et al. Prevalence of chronic obstructive pulmonary disease and associated risk factors in Uganda (FRESH AIR Uganda): a prospective cross-sectional observational study. Lancet Glob Health. 2015;3:e44-51. Medline:25539969 doi:10.1016/S2214-109X(14)70337-7

61 Aggarwal AN, Gupta D, Agarwal R, Jindal SK. Comparison of the Lower Confidence Limit to the Fixed-Percentage Method for Assessing Airway Obstruction in Routine Clinical Practice. Respir Care. 2011;56:1778-84. Medline:21605485 doi:10.4187/ respcare. 01160

62 Hnizdo E, Glindmeyer HW, Petsonk EL, Enright P, Buist AS. Case definitions for chronic obstructive pulmonary disease. COPD. 2006;3:95-100. Medline:17175672 doi:10.1080/15412550600651552

63 Szanto O, Montnemery P, Elmstahl S. Prevalence of airway obstruction in the elderly: results from a cross-sectional spirometric study of nine age cohorts between the ages of 60 and 93 years. Prim Care Respir J. 2010;19:231-6. Medline:20228994 doi:10.4104/pcrj.2010.00011

64 Celli BR, MacNee W, Agusti A, Anzueto A, Berg B, Buist AS, et al. Standards for the diagnosis and treatment of patients with COPD: a summary of the ATS/ERS position paper. Eur Respir J. 2004:23:932-46. Medline:15219010 doi:10.1183/0903193 6.04.00014304

65 Agarwal D, Parker R, Pinnock H, Roy S, Ghorpade D, Salvi S, et al. Normal spirometry predictive values for the Western Indian adult population. Eur Respir J. 2020;56:1902129. Medline:32366494 doi:10.1183/13993003.02129-2019

66 Obaseki DO, Erhabor GE, Gnatiuc L, Adewole OO, Buist SA, Burney PG. Chronic Airflow Obstruction in a Black African Population: Results of BOLD Study, Ile-Ife, Nigeria. COPD. 2016;13:42-9. Medline:26451840 doi:10.3109/15412555.2015 .1041102

67 Johannessen A, Omenaas ER, Bakke PS, Gulsvik A. Implications of reversibility testing on prevalence and risk factors for chronic obstructive pulmonary disease: a community study. Thorax. 2005;60:842-7. Medline:16085729 doi:10.1136/thx.2005.043943

68 Bush A, Kleinert S, Pavord ID. The asthmas in 2015 and beyond: a Lancet Commission. Lancet. 2015;385:1273-5. Medline:25890896 doi:10.1016/S0140-6736(15)60654-7

69 White J, Paton JY, Niven R, Pinnock H; British Thoracic Society. Guidelines for the diagnosis and management of asthma: a look at the key differences between BTS/SIGN and NICE. Thorax. 2018;73:293-7. doi:10.1136/thoraxjnl-2017-211189 